\title{
Erratum to: Analytical solution of Bohr Hamiltonian and extended form of sextic potential using bi-confluent Heun functions
}

\author{
H. Sobhani ${ }^{1, a}$, A.N. Ikot ${ }^{2}$, and H. Hassanabadi ${ }^{1, b}$ \\ 1 Physics Department, Shahrood University of Technology, P. O. Box: 3619995161-316 Shahrood, Iran \\ 2 Theoretical Physics Group, Department of Physics, University of Port Harcourt, Choba PMB 5323 Port Harcourt, Nigeria
}

Original article: Eur. Phys. J. Plus (2017) 132: 240, https://doi.org/10.1140/epjp/i2017-11493-9

Received: 6 July 2018 / Revised: 27 August 2018

Published online: 18 October 2018

(c) Società Italiana di Fisica / Springer-Verlag GmbH Germany, part of Springer Nature, 2018

After publication of our paper [1], we noticed an error for the energy and the wave function. We evaluate the numerical results once again. We can find more accurate numerical results and the physical interpretations do not change.

\section{The energy and the wave function}

The solution of eq. (14) of our paper [1] can be written as

$$
\begin{aligned}
F_{L, \alpha} & =H_{b}\left(\alpha^{\prime}, \beta^{\prime}, \delta^{\prime}, \gamma^{\prime} ; \beta^{2}\right), \\
\alpha^{\prime} & =\sqrt{W+a+\frac{9}{4}}, \\
\beta^{\prime} & =\frac{c}{4} \\
\gamma^{\prime} & =\frac{1}{64}\left(-16 b+c^{2}\right), \\
\delta^{\prime} & =\frac{-\varepsilon_{\beta}}{2},
\end{aligned}
$$

where we have corrected the expression for $\gamma^{\prime}$. Therefore, the new expressions for energy and constraint are obtained as

$$
\begin{aligned}
\varepsilon_{\beta} & =\frac{c}{2}\left(\sqrt{a+W+\frac{9}{4}}+2 n+5\right), \\
b & =\frac{c^{2}}{16}-4\left(\sqrt{a+W+\frac{9}{4}}+2 n+4\right) .
\end{aligned}
$$

According to the corrected results, we shall evaluate the numerical evaluations presented in our paper once again.

\section{Numerical results}

In this section, according to the expressions for energy and constraint, we evaluate the theoretical predictions in tables $1-4$.

\footnotetext{
a e-mail: hadisobhani8637@gmail.com (corresponding author)

b e-mail: hha1349@gmail.com
} 
Table 1. Comparison of our results with experimental data for three isotopes of xenon.

\begin{tabular}{ccccccc}
\hline Isotope & \multicolumn{2}{c}{${ }^{128} \mathrm{Xe}$} & \multicolumn{2}{c}{${ }^{130} \mathrm{Xe}$} & \multicolumn{2}{c}{${ }^{132} \mathrm{Xe}$} \\
\hline$L_{\text {band }}$ & Theor. & Exp. & Theor. & Exp. & Theor. & Exp. \\
\hline $4_{g}$ & 2.323 & 2.333 & 2.255 & 2.247 & 2.150 & 2.157 \\
$6_{g}$ & 3.806 & 3.922 & 3.619 & 3.627 & 3.353 & 3.163 \\
$8_{g}$ & 5.373 & 5.627 & 5.037 & 5.031 & & \\
$10_{g}$ & 6.988 & 7.597 & 6.484 & 6.457 & & \\
$12_{g}$ & & & 7.949 & 7.867 & & \\
$14_{g}$ & & & 9.426 & 9.458 & & \\
\hline $2_{\gamma}$ & 1.830 & 2.189 & 1.793 & 2.093 & 1.734 & 1.944 \\
$3_{\gamma}$ & 2.556 & 3.228 & 2.471 & 3.045 & 2.343 & 2.701 \\
$4_{\gamma}$ & 4.180 & 3.620 & 3.959 & 3.373 & 3.649 & 2.940 \\
$5_{\gamma}$ & 4.361 & 4.508 & 4.123 & 4.051 & 3.791 & 3.246 \\
$6_{\gamma}$ & 6.286 & 5.150 & & & & \\
$7_{\gamma}$ & 6.139 & 6.165 & & & & \\
\hline $0_{\beta}$ & 3.454 & 3.574 & 3.025 & 3.346 & 2.528 & 2.771 \\
$2_{\beta}$ & 4.454 & 4.515 & & & & \\
\hline$a$ & 3.043 & & 1.506 & & 0 & \\
\hline$\sigma$ & 0.493 & & 0.297 & & 0.422 & \\
\hline & & & & & & \\
\hline
\end{tabular}

Table 2. Comparison of our results with experimental data for three isotopes of platinum.

\begin{tabular}{ccccccc}
\hline Isotope & \multicolumn{2}{c}{${ }^{192} \mathrm{Pt}$} & \multicolumn{2}{c}{${ }^{194} \mathrm{Pt}$} & \multicolumn{2}{c}{${ }^{196} \mathrm{Pt}$} \\
\hline$L_{\text {band }}$ & Theor. & Exp. & Theor. & Exp. & Theor. & Exp. \\
\hline $4_{g}$ & 2.391 & 2.479 & 2.410 & 2.470 & 2.388 & 2.465 \\
$6_{g}$ & 4.004 & 4.314 & 4.060 & 4.298 & 3.994 & 4.290 \\
$8_{g}$ & 5.743 & 6.377 & 5.852 & 6.392 & 5.725 & 6.333 \\
$1_{g}$ & 7.559 & 8.624 & 7.730 & 8.672 & 7.530 & 8.558 \\
\hline $2_{\gamma}$ & 1.866 & 1.935 & 1.876 & 1.894 & 1.864 & 1.936 \\
$3_{\gamma}$ & 2.641 & 2.910 & 2.664 & 2.809 & 2.637 & 2.825 \\
$4_{\gamma}$ & 4.416 & 3.795 & 4.484 & 3.743 & 4.405 & 3.636 \\
$5_{\gamma}$ & 4.617 & 4.682 & 4.690 & 4.563 & 4.604 & 4.526 \\
$6_{\gamma}$ & 6.767 & 5.905 & & & 6.743 & 7.730 \\
$7_{\gamma}$ & 6.602 & 6.677 & & & & \\
\hline $0_{\beta}$ & 4.012 & 3.776 & 4.197 & 3.858 & 3.981 & 3.192 \\
$2_{\beta}$ & 5.012 & 4.547 & 5.197 & 4.603 & 4.981 & 3.828 \\
\hline$a$ & 5.364 & & 6.217 & & 5.228 & \\
\hline$\sigma$ & 0.533 & & 0.505 & & 0.713 & \\
\hline
\end{tabular}


Table 3. Normalized $B(E 2)$ transition rats for ${ }^{128} \mathrm{Xe}$ and ${ }^{132} \mathrm{Xe}$.

\begin{tabular}{cccccc}
\hline \multicolumn{5}{c}{${ }^{128} \mathrm{Xe}$} & \multicolumn{2}{c}{${ }^{132} \mathrm{Xe}$} \\
\hline$L_{\text {band }}^{(i)}$ & $L_{\text {band }}^{(f)}$ & Theor. & Exp. & Theor. & Exp. \\
\hline $4_{g}$ & $2_{g}$ & 1.512 & 1.468 & 1.596 & 1.238 \\
$6_{g}$ & $4_{g}$ & 2.065 & 1.940 & & \\
$8_{g}$ & $6_{g}$ & 2.483 & 2.388 & & \\
$2_{\gamma}$ & $2_{g}$ & 1.546 & 1.194 & 1.632 & 1.775 \\
$2_{\gamma}$ & $0_{g}$ & 0 & 0.016 & 0 & 0.003 \\
\hline$c$ & \multicolumn{7}{c}{0} \\
\hline$\sigma$ & 0.166 & & & \\
\hline
\end{tabular}

Table 4. Normalized $B(E 2)$ transition rats for ${ }^{192} \mathrm{Pt},{ }^{194} \mathrm{Pt}$ and ${ }^{196} \mathrm{Pt}$.

\begin{tabular}{cccccccc}
\hline & \multicolumn{3}{c}{${ }^{192} \mathrm{Pt}$} & \multicolumn{2}{c}{${ }^{194} \mathrm{Pt}$} & \multicolumn{2}{c}{${ }^{196} \mathrm{Pt}$} \\
\hline$L_{\text {band }}^{(i)}$ & $L_{\text {band }}^{(f)}$ & Theor. & Exp. & Theor. & Exp. & Theor. & Exp. \\
\hline $4_{g}$ & $2_{g}$ & 1.486 & 1.559 & 1.479 & 1.724 & 1.487 & 1.476 \\
\hline $4_{\gamma}$ & $2_{\gamma}$ & & & 0.629 & 0.446 & 0.670 & 0.715 \\
$6_{\gamma}$ & $4_{\gamma}$ & & & & & 0.933 & 1.208 \\
\hline $3_{\gamma}$ & $2_{\gamma}$ & 1.064 & 1.786 & & & & \\
\hline $2_{\gamma}$ & $0_{g}$ & 0 & 0.009 & 0 & 0.006 & 0 & 0.0004 \\
$2_{\gamma}$ & $2_{g}$ & 1.518 & 1.909 & 1.512 & 1.805 & & \\
$4_{\gamma}$ & $2_{g}$ & & & 0 & 0.004 & 0 & 0.014 \\
$4_{\gamma}$ & $4_{g}$ & & & 0.313 & 0.406 & & \\
$6_{\gamma}$ & $4_{g}$ & & & & & 0 & 0.012 \\
\hline$c$ & & 300 & & 300 & & 300 & \\
\hline$\sigma$ & & 0.476 & & 0.194 & & 0.125 & \\
\hline
\end{tabular}

\section{References}

1. H. Sobhani, A.N. Ikot, H. Hassanabadi, Eur. Phys. J. Plus 132, 240 (2017). 